\title{
Overproduction of the Pro-Apoptotic Molecule, Programmed Cell Death 5, in Toxoplasma gondii Leads to Increased Apoptosis of Host Macrophages
}

\author{
Hiroshi BANNAI ${ }^{1}$, Yoshifumi NISHIKAWA ${ }^{1) *}$, Hany Mohamed IBRAHIM ${ }^{1)}$, Kyoko YAMADA ${ }^{1)}$, Osamu KAWASE ${ }^{1)}$, \\ Jun-ichi WATANABE ${ }^{2)}$, Chihiro SUGIMOTO ${ }^{3)}$ and Xuenan XUAN ${ }^{1)}$ \\ ${ }^{1)}$ National Research Center for Protozoan Diseases, Obihiro University of Agriculture and Veterinary Medicine, Inada-cho, Obihiro, \\ Hokkaido 080-8555, ${ }^{2)}$ The Institute of Medical Science, The University of Tokyo, Shirokanedai, Minato-ku, Tokyo 108-8639 and \\ ${ }^{3)}$ Research Center for Zoonosis Control, Hokkaido University, Chuou-ku, Sapporo 060-0818, Japan
}

(Received 22 March 2009/Accepted 17 May 2009)

ABSTRACT. We established a recombinant strain of Toxoplasma gondii that overexpressed programmed cell death 5 (TgPDCD5), in order to evaluate the role of endogenous TgPDCD5 in macrophage apoptosis during $T$. gondii infection. Immunofluorescence microscopy revealed that overproduced TgPDCD5 with a hemagglutinin tag was localized in the cytosol, which was consistent with the localization of endogenous TgPDCD5. The induced TgPDCD5-HA was recognized as an additional band by Western blot analysis, indicating successful overexpression of TgPDCD5. Secretion and release of TgPDCD5 by the parasite was also up-regulated in a time-dependent manner, which reflected its overproduction. Apoptosis due to parasite infection and interferon-gamma treatment was significantly upregulated by the overexpression of TgPDCD5. These results suggest that endogenous TgPDCD5 plays a role in macrophage apoptosis during $T$. gondii infection.

KEY WORDS: apoptosis, overexpression, PDCD5, Toxoplasma gondii.

Toxoplasma gondii is an obligate intracellular parasite that can infect virtually all species of warm-blooded animals, including humans, worldwide. Domestic cats and other felines are the definitive hosts, while non-feline hosts act as intermediate hosts [3]. T. gondii infection is usually asymptomatic in immunocompetent hosts, and severe cases occur only rarely. However, it remains a serious threat to human health due to its high prevalence, which is typically $10-30 \%$ in most populations, but sometimes even higher [33]. In addition to causing life-threatening encephalitis in immuno-compromised hosts such as AIDS patients, T. gondii can also cause miscarriage, stillbirth, or fatal birth defects in humans if contracted during pregnancy [23, 29].

Acute infection of both humans and mice with $T$. gondii induces as state of transient immunosuppression, characterized by reduced antibody and T lymphocyte responses [22, $32,38,39]$. In addition, the apoptosis of $\mathrm{T}$ lymphocytes triggered by $T$. gondii has been thought to restrict the immune response to the parasite $[14,17,36]$. Indeed, high levels of apoptosis in splenocytes have been associated with unrestricted parasite multiplication leading to high parasite burdens in various host tissues [6, 24]. Apoptosis of peritoneal macrophages has also been reported in infected mice $[11,25]$. Apoptosis in macrophages was also confirmed in an in vitro study, which showed that $T$. gondii infection of J774A.1 mouse macrophage cells treated with interferongamma (IFN- $\gamma)$ resulted in activation of inducible nitric

\footnotetext{
* Correspondence to: Nishikawa, Y., National Research Center for Protozoan Diseases, Obihiro University of Agriculture and Veterinary Medicine, Inada-cho, Obihiro, Hokkaido 080-8555, Japan.

e-mail: nisikawa@obihiro.ac.jp
}

oxide synthase, and that the consequent production of nitric oxide facilitated apoptosis [25]. This immune cell apoptosis is thought to lead to immunosuppression, and so contribute to parasite survival in the host.

T. gondii programmed cell death 5 (TgPDCD5) was identified as a homologue of the human apoptosis-related molecule PDCD5 [1, 2, 18, 30, 34, 35] and the Plasmodium falciparum apoptosis-related protein [9]. We reported that recombinant TgPDCD5 (rTgPDCD5) exerted an apoptosisenhancing effect on host cells in the presence of the topoisomerase II inhibitor, etoposide, or in cooperation with IFN$\gamma$ [1]. The cell-penetration activity of rTgPDCD5 via a heparan-sulfate proteoglycan (HSPG)-binding motif was confirmed to be highly associated with its pro-apoptotic effect, as observed in the human PDCD5 protein [1, 34]. Endogenous TgPDCD5 is a secreted protein, despite having no signal peptide, suggesting that its secretion occurs not via the typical pathway seen in microneme proteins or rhoptry proteins, but via an unknown mechanism. This is a common feature of previously described translocatory proteins with highly basic regions such as human immunodeficiency virus Tat [5, 8], Drosophila antennapedia [12], and herpes simplex virus VP22 [4]. These proteins are known to penetrate into the cells via HSPG on the surface membrane, and this fact can be applied for the development of drug delivery tools.

In order to clarify the role of endogenous TgPDCD5 in host cell apoptosis during $T$. gondii infection, we established a recombinant parasite that overexpressed TgPDCD5. We aimed to clarify the role of endogenous TgPDCD5 in host macrophages and establish whether or not it showed similar apoptosis-enhancing activity to the recombinant protein. 


\section{MATERIALS AND METHODS}

Cell lines: The mammalian cell lines used in this study were chinese hamster ovary epithelial cells (CHO-K1, American Type Culture Collection [ATCC] CCL-61), mouse macrophages (J774A.1, ATCC TIB-67), and monkey kidney adherent fibroblasts (Vero, ATCC CCL-81). Cells were maintained as described in the ATCC instructions. T. gondii isolates used were PLK wild-type strain (PLK-WT), a recombinant PLK strain that expressed green fluorescent protein (GFP) (PLK-GFP) [27], and recombinant PLK strains that overexpressed TgPDCD5 (PLK-TgPDCD5) or $\Delta 106-122$ (PLK- $\Delta 106$ ) (see below). The parasite stock was maintained in vitro by serial passages in a monolayer of CHO-K1 cells.

Production of anti-GST-TgPDCD5 serum and purification of $\operatorname{IgG}$ : rTgPDCD5 fused with glutathione S-transferase (GST) was expressed in Escherichia coli, and antiGST-TgPDCD5 serum from mice and a rabbit were produced as described previously [1]. Animals used in this study were cared for and used under the Guiding Principles for the Care and Use of Research Animals promulgated by the Obihiro University of Agriculture and Veterinary Medicine.

Construction of transfer vector: The plasmid for overexpression in $T$. gondii, pDMG was constructed to express GFP, dihydrofolate reductase-thymidylate synthase (DHFR-TS), and the target protein [26]. The DNA fragment for full-length TgPDCD5 was amplified by polymerase chain reaction from the full-length cDNA clone XTG05779 of Full-Toxo [13] using the primers 5'-ATC CAT GGA TGC AGC CTG AAG AAT TCG CC-3' and 5'TAG CTA GCG AAG TCA TCG TCG TCG TCA CT-3'. For $\Delta 106-122$, which was truncated 17 amino acids from the C-terminus, the reverse primer 5'-TAG CTA GCA TTC TTC GCC GCA GAC GCC GC-3' was used. The resulting products were digested with $\mathrm{NcoI}$ and $\mathrm{NheI}$ and inserted into the same sites of pHXNTPHA (kindly provided by K.A. Joiner, Yale University), to produce pHXNTP-TgPDCD5 (or $\Delta 106-122$ )-hemagglutinin (HA). TgPDCD5 (or $\Delta 106-$ 122) with a HA tag (YPYDVPDYA) was obtained from pHXNTP-TgPDCD5 (or $\Delta 106-122$ )-HA by NcoI and BglII digestion. This fragment was blunt ended by Klenow fragment and inserted into the EcoRV site of pDMG; pDMGTgPDCD5 and pDMG- $\Delta 106$, which contained expression cassettes of GFP and DHFR-TS, and TgPDCD5-HA (or $\Delta$ 106-122-HA) were obtained.

Transfection and selection of $T$. gondii-overexpressing TgPDCD5 or 4106-122: Electroporation of tachyzoites was performed as described previously [31]. In brief, purified $T$. gondii PLK tachyzoites were resuspended at $10^{7}$ cell $/ \mathrm{m} l$ with cytomix buffer $\left(120 \mathrm{mM} \mathrm{KCl}, 0.15 \mathrm{mM} \mathrm{CaCl}_{2}, 10 \mathrm{mM}\right.$ $\mathrm{K}_{2} \mathrm{HPO}_{4}-\mathrm{KH}_{2} \mathrm{PO}_{4}, 2 \mathrm{mM}$ EDTA, $5 \mathrm{mM} \mathrm{MgCl} 2,25 \mathrm{mM}$ HEPES [pH 7.6]), supplemented with $2 \mathrm{mM}$ ATP and $5 \mathrm{mM}$ glutathione. Cells were transferred to a 2-mm-gap cuvette and electroporated with $2.0 \mathrm{keV}$ at $50 \mathrm{~W}$ using a Gene Pulser II (Bio-Rad Laboratories, Hercules, U.S.A.). After transfection, tachyzoites were allowed to infect Vero cells in drug-free culture medium for $18 \mathrm{hr}$ to permit the phenotypic expression of the DHFR-TS gene and GFP selectable markers, after which pyrimethamine was added to a final concentration of $1 \mu \mathrm{M}$. Polyclonal transfected, pyrimethamineresistant tachyzoite cultures were subjected to plaque purification. The cultures were passaged three times in medium containing $1 \%$ agarose, and a single plaque was obtained. The recombinant $T$. gondii clones constructed using transfer vectors, pDMG-TgPDCD5 and pDMG- $\triangle 106$ were designated as PLK-TgPDCD5 and PLK- $\Delta 106$, respectively.

Indirect fluorescent antibody test (IFAT): Confluent CHO-K1 cells on coverslips were inoculated with PLKTgPDCD5 or PLK- $\Delta 106$. The coverslips were collected at $24 \mathrm{hr}$ post-parasite inoculation, washed, fixed and then subjected to IFAT as described previously [1]. Briefly, the coverslips were treated with anti-HA monoclonal antibody (Covance Research Products, Inc., Berkeley, U.S.A.) as primary antibody diluted at 1:500 in 3\% bovine serum albumin (BSA) in phosphate-buffered saline (PBS) containing $1 \mathrm{mM}$ $\mathrm{CaCl}_{2}$ and $\mathrm{MgCl}_{2}$ (PBS++) for $1 \mathrm{hr}$ at room temperature. After washing three times with $\mathrm{PBS}++$, the coverslips were incubated with Alexa Fluor 594-conjugated goat anti-mouse IgG (Invitrogen, Carlsbad, U.S.A.) as secondary antibody diluted at 1:600 in 3\% BSA in PBS++ for $1 \mathrm{hr}$ at room temperature and then washed again. For the control staining of native TgPDCD5 in PLK-WT, infected cells were fixed and immunostained with mouse anti-rTgPDCD5 sera and Alexa Fluor 488-conjugated goat anti-mouse IgG. Dilutions of primary and secondary antibodies were 1:100 and 1:600, respectively. The coverslips were placed on a glass slide coated with Mowiol (EMD Biosciences, Inc., La Jolla, U.S.A.). The slides were examined by confocal laser scanning microscopy (TCS-NT, Leica Microsystems GmbH, Wetzlar, Germany).

Western blot analysis of PLK-TgPDCD5 and PLK- 4106 : The parasite lysate was prepared according to the method described previously [1]. Briefly, $1 \times 10^{8}$ tachyzoites of PLK-WT, PLK-TgPDCD5 or PLK- $\Delta 106$ strains were harvested, lysed and sonicated. Protein samples were electrophoresed in each lane of a $15 \%$ polyacrylamide gel and transferred onto a nitrocellulose membrane (Whatman GmbH, Dassel, Germany). After washing twice with a washing solution, the membrane was incubated with 5\% skimmed milk in PBS for $30 \mathrm{~min}$ at room temperature. After two further washes, the membrane was incubated with anti-rTgPDCD5 mouse serum, anti-HA mouse monoclonal antibody, or anti-SAG1 mouse monoclonal antibody (Advanced Immunochemical, Inc., Long Beach, U.S.A.) for $1 \mathrm{hr}$ at room temperature. The dilutions of primary antibodies were 1:100 for anti-rTgPDCD5 mouse serum, 1:1,000 for anti-HA antibody, and 1:500 for anti-SAG1 antibody. After washing three times, the membranes were incubated with horseradish peroxidase (HRP)-conjugated goat antimouse IgG antibodies (Bethyl, Inc., Montgomery, U.S.A.) diluted at 1:8,000 in $0.5 \%$ skimmed milk in PBS for $1 \mathrm{hr}$ at room temperature. After washing three times, the proteins 
were visualized on X-ray film using the ECL Detection Reagents (GE Healthcare UK Ltd., Buckinghamshire, UK) according to the manufacturer's recommendations.

Sandwich enzyme-linked immunosorbent assay (ELISA) for the detection of secreted TgPDCD5: In order to detect secreted TgPDCD5 from extracellular parasites, purified $T$. gondii tachyzoites of PLK-WT, PLK-GFP, PLK-TgPDCD5 and PLK- $\Delta 106\left(5 \times 10^{6}\right)$ were incubated in $100 \mu l$ of GIT medium (Nihon Pharmaceutical Co., Ltd., Tokyo, Japan) at $37^{\circ} \mathrm{C}$ for 15 or $30 \mathrm{~min}$. The culture supernatant at each time point was collected by sequential centrifugation $(500 \times \mathrm{g}$ for 10 min at $4{ }^{\circ} \mathrm{C}$, then $8,400 \times \mathrm{g}$ for $10 \mathrm{~min}$ at $4^{\circ} \mathrm{C}$ ), and subjected to sandwich ELISA without condensation.

Rabbit anti-rTgPDCD5 polyclonal immunoglobulin-G ( $\operatorname{IgG}$ ) was purified using Protein A chromatography columns according to the manufacturer's instructions (Bio-Rad Laboratories). Protein concentrations were measured using a BCA protein assay kit (Thermo Fisher Scientific, Inc., Rockford, U.S.A.). One microgram of the IgG diluted in a $0.05 \mathrm{M}$ carbonate buffer ( $\mathrm{pH}$ 9.6) was used as the capture antibody to coat microtiter plates at $4^{\circ} \mathrm{C}$ overnight. Blocking was performed with a blocking solution ( $3 \%$ skimmed milk in PBS, $\mathrm{pH} 7.2$ ) at $37^{\circ} \mathrm{C}$ for $2 \mathrm{hr}$. The plates were incubated at $37^{\circ} \mathrm{C}$ for $30 \mathrm{~min}$ with each concentrated supernatant. After washing six times with a washing solution (0.05\% Tween 20 in PBS), anti-rTgPDCD5 mouse serum diluted at 1:100 in a blocking solution was added to each well as a detection antibody. After a further 6 washes, the plates were incubated with HRP-conjugated goat antimouse IgG antibodies (Bethyl, Inc.) diluted at 1:2,500 in a blocking solution. Binding was visualized using a substrate solution $\quad\left(0.3 \mathrm{mg} / \mathrm{ml} 2,2^{\prime}\right.$-azino-bis-(3-ethlbenz-thiazoline-6-sulfonic acid), $0.1 \mathrm{M}$ citric acid, $0.2 \mathrm{M}$ sodium phosphate, $0.003 \% \mathrm{H}_{2} \mathrm{O}_{2}$ ). The absorbance at $415 \mathrm{~nm}$ was measured using a MTP-500 microplate reader (Corona Electric, Tokyo, Japan). The TgPDCD5 concentration of each sample was calculated by standardization with rTgPDCD5.

Apoptosis assay: J774A.1 cells $\left(2.5 \times 10^{5}\right)$ were inoculated with $T$. gondii PLK-WT, PLK-GFP, PLK-TgPDCD5 or PLK- $\Delta 106\left(5 \times 10^{5}\right)$ and then incubated with or without $20 \mathrm{U} / \mathrm{m} l$ of IFN- $\gamma$ (Thermo Fisher Scientific, Inc.) for $24 \mathrm{hr}$ at $37^{\circ} \mathrm{C}$. Cells were scraped, washed with PBS, re-suspended in PBS, and placed on glass slides. The slides were dried and then fixed, permeabilized and then stained with an In Situ Cell Death Detection Kit, TMR red (Roche Diagnostics $\mathrm{GmbH}$, Mannheim, Germany) to visualize apoptotic cells using the terminal deoxynucleotidyltransferase-mediated dUTP-biotin nick-end labeling (TUNEL) method, as described previously [1]. The slides were examined using a fluorescence microscope (Nikon, Tokyo, Japan). The mean ratio of apoptotic cells was calculated as the number of TUNEL-positive cells divided by the total number of cells $x$ 100 , in three individual fields from each sample. Each field contained at least 300 cells. Statistical significance was calculated using Student's $t$-test based on the mean and S.D. of triplicate samples.

\section{RESULTS}

Construction and characterization of $T$. gondii overexpressing TgPDCD5: A recombinant parasite overexpressing TgPDCD5 with an HA tag was established to confirm the effect of high levels of TgPDCD5 on host cell apoptosis. Two independent clones were isolated from the transfected polyclonal culture and were designated as PLK-TgPDCD51 and -2 . The reactivity of the recombinant parasites to the anti-HA monoclonal antibody and the fluorescence of GFP were confirmed by IFAT (Fig. 1A). The cytosolic localization of TgPDCD5-HA was consistent with that of endogenous TgPDCD5 (Fig. 1A).

Western blot analysis was performed to confirm the expression of endogenous and induced TgPDCD5. An antiSAG1 antibody was used as a control to confirm that each lane contained the same amount of parasite lysate. Western blotting with anti-rTgPDCD5 sera indicated that PLKTgPDCD5 clones expressed additional bands of a slightly larger size $(15.5 \mathrm{kDa})$ than that of the endogenous protein (14.5 kDa) (Fig. 1B). The expression of induced TgPDCD5-HA was also confirmed in the anti-HA antibodytreated blot, although the existence of two bands in each lane was not expected (Fig. 1B). The upper band was consistent with that observed as the additional band in the antirTgPDCD5 blot while the lower one possibly represented a degraded product. A recombinant parasite overexpressing $\Delta$ 106-122-HA, which lacks the HSPG-binding motif, was also established and designated as PLK- $\Delta 106$. The expression of induced $\Delta 106-122$-HA was confirmed by IFAT as in PLK-TgPDCD5 (Fig. 1A). The single band ( $14.5 \mathrm{kDa})$ seen in the PLK- $\Delta 106$ lysate treated with anti-rTgPDCD5 serum by Western blot may be due to the similar sizes of native TgPDCD5 and $\triangle 106-122-H A$ (Fig. 1B). Two bands were present in the anti-HA antibody-treated blot, as observed for PLK-TgPDCD5. The upper band was consistent with the predicted size of $\triangle 106-122-\mathrm{HA}(14.5 \mathrm{kDa})$, while the lower one possibly represented a degraded product. An HA tag was fused with C-terminus of TgPDCD5 or $\Delta 106-102$. Therefore, lower band detected by anti-HA antibody may be a product cleaved about 9 amino acids from the $\mathrm{N}$-terminus of the intact TgPDCD5 or $\triangle 106-102$. However, no domain was predicted to be the signal sequence in TgPDCD5 while this molecule was secreted protein [1]. Since only one band was detected in PLK-WT using anti-TgPDCD5 antibody, degraded products seen using anti-HA antibody might be artifact.

Increased levels of TgPDCD5 secreted/released into the supernatant of PLK-TgPDCD5 and parasitized cells: The secretion of TgPDCD5 by the extracellular parasite and its dependency on incubation time was confirmed in our previous study [1]. The overproduction of full-length and truncated TgPDCD5, in addition to the native protein, was confirmed by Western blot analysis (Fig. 1B), and the secretion of TgPDCD5 from the recombinant parasites was measured using a sandwich ELISA. The secretion of TgPDCD5 from PLK-WT was slightly increased to $126.1 \pm 12.9 \mathrm{pg} / \mathrm{ml}$ 


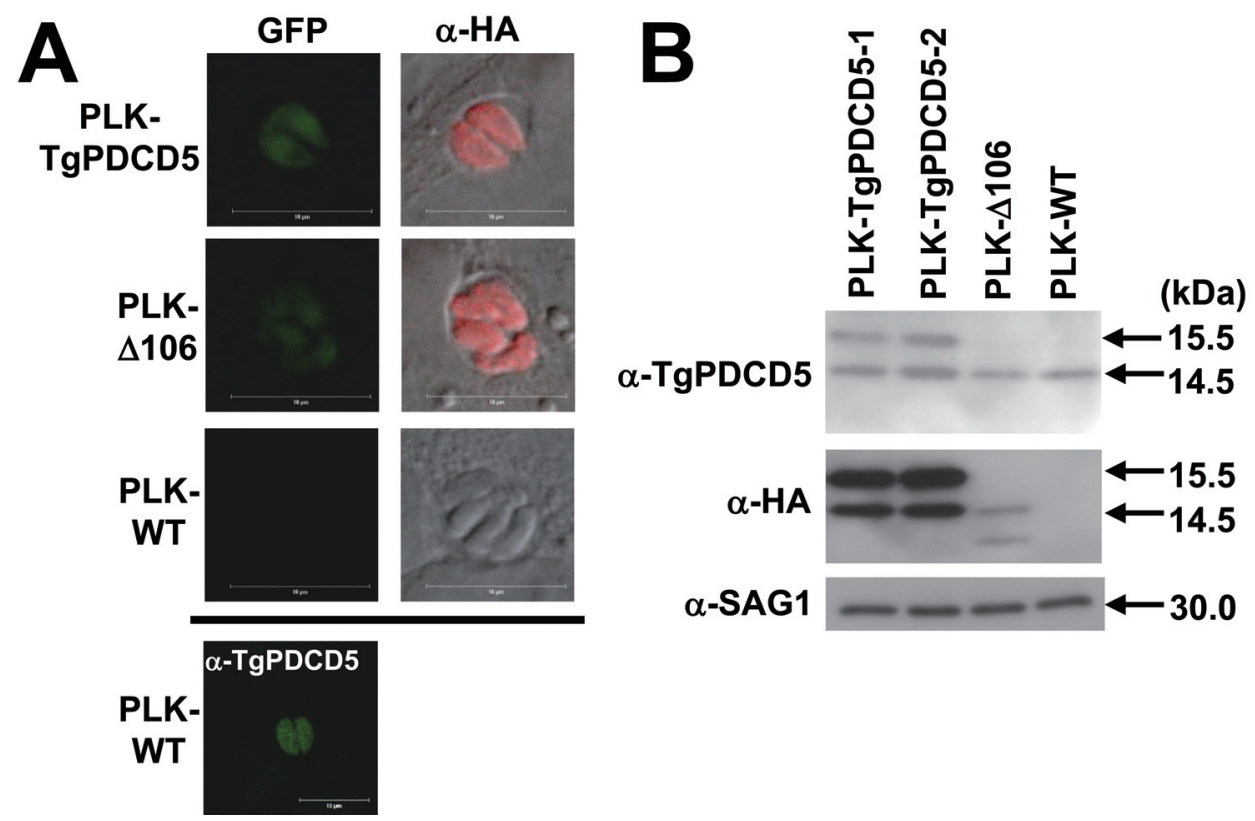

Fig. 1. Expression of TgPDCD5 with hemagglutinin (HA) tag in PLK-TgPDCD5 and PLK- $\Delta 106$. (A) Indirect fluorescent antibody test images of PLK-TgPDCD5, PLK- $\triangle 106$, and PLK-WT. T. gondiiinfected CHO-K1 cells on coverslips were fixed and immunostained with mouse anti-HA monoclonal antibody and Alexa Fluor 594-conjugated goat anti-mouse IgG. For the control staining of native TgPDCD5 in PLK-WT, infected cells were fixed and immunostained with mouse anti-rTgPDCD5 sera and Alexa Fluor 488-conjugated goat anti-mouse IgG. Bar, $10 \mu \mathrm{m}$. (B) Western blot analysis of PLKTgPDCD5 and PLK- $\Delta 106$. The lysates from recombinant and wild-type parasites were electrophoresed in a $15 \%$ polyacrylamide gel and transferred onto a nitrocellulose membrane. This was then probed with the mouse anti-rTgPDCD5 antibody, mouse anti-HA antibody, or mouse anti-SAG1 monoclonal antibody, followed by horseradish peroxidase-conjugated goat anti-mouse $\operatorname{IgG}$ antibody.

at $30 \mathrm{~min}$ (Fig. 2A). However, PLK-TgPDCD5-1 (246.1 \pm $20.1 \mathrm{pg} / \mathrm{ml}, P<0.01)$, PLK-TgPDCD5-2 (228.6 $\pm 22.0 \mathrm{pg} /$ $\mathrm{ml}, P<0.01)$, and PLK- $\Delta 106(194.9 \pm 32.0 \mathrm{pg} / \mathrm{ml}, P<0.05)$ showed significantly higher levels of TgPDCD5.

Evaluation of the effects of PLK-TgPDCD5 on macrophage apoptosis in the presence of IFN- $\gamma$ : The effect of different concentration of rTgPDCD5 on host cell apoptosis has been confirmed in our previous study, indicating dosedependency of the pro-apoptotic effect [1]. In this experiment, PLK-TgPDCD5-1, PLK-TgPDCD5-2, PLK- $\Delta 106$, PLK-WT, and PLK-GFP parasites were inoculated into J774A. 1 cells and treated with IFN- $\gamma$. The ratios of apoptotic cells observed in the PLK-TgPDCD5-1- and PLKTgPDCD5-2-infected groups were $23.71 \pm 1.11 \%(P<0.01)$ and $23.40 \pm 2.06 \%(P<0.01)$, respectively. These were significantly higher than those in the PLK- $\Delta 106-(9.44 \pm$ $1.07 \%)$, PLK-WT- $(9.45 \pm 1.02 \%)$, and PLK-GFP-infected $(9.38 \pm 0.58 \%)$ groups (Fig. 3$)$. This result strongly suggests that parasite-expressed TgPDCD5 was active in apoptosis induction, and that an increased level of TgPDCD5 protein was linked to the high rate of host cell apoptosis.

\section{DISCUSSION}

Many factors have been suggested to contribute to T. gon- dii's ability to establish and maintain a persistent infection in immunocompetent hosts. Several reports have indicated that this includes alterations in apoptosis in distinct host cell populations $[10,20]$. This is not surprising because apoptosis is known to play a critical role in the regulation of the immune response [28], as an effector mechanism whereby, natural killer (NK) cells and cytotoxic T lymphocytes eliminate infected cells [16], and as an innate response of cells after infection by intracellular pathogens [37]. More interestingly, T. gondii both promotes and inhibits apoptosis [19]. The inhibition of host cell apoptosis may allow undisturbed intracellular development, thereby facilitating parasite survival. Increased apoptosis of immune cells after infection, on the other hand, is thought to partially disturb the host immune responses to $T$. gondii, leading to immune evasion.

T. gondii actively down-regulates the expression of major histocompatibility complex class I and class II molecules in murine macrophages and monocytes, which may directly inhibit their antigen-presenting capacity $[15,21]$. Apoptosis caused by parasite infection in splenocytes, including $\mathrm{CD}^{+}$ and $\mathrm{CD}^{+}$lymphocytes, B lymphocytes, NK cells, and granulocytes, and in Peyer's patch T cells, may also contribute to suppression of the immune response [7, 17]. Likewise, the apoptosis observed in macrophages is also considered to be 


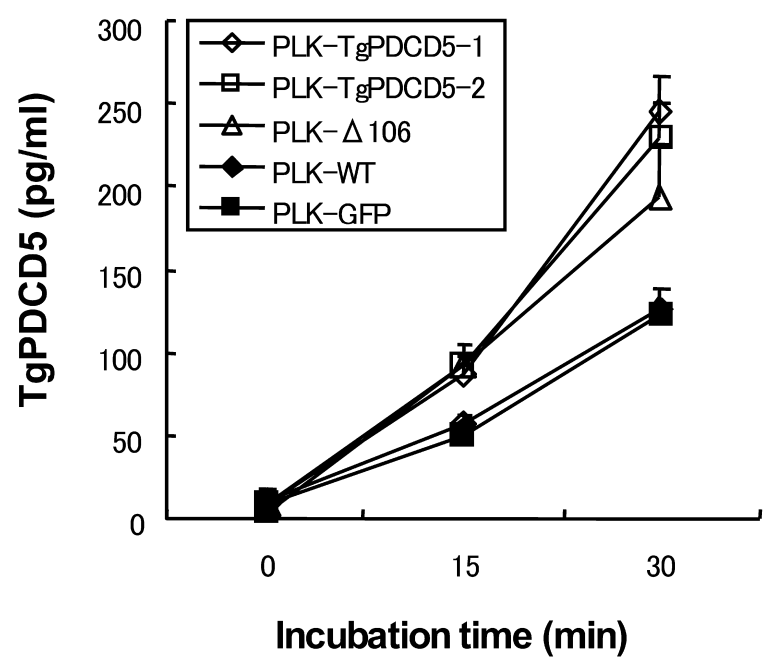

Fig. 2. TgPDCD5 detected in the culture medium of free parasites or parasite-infected cells. The purified tachyzoites of PLK-TgPDCD5-1, PLK-TgPDCD5-2, PLK- 106 , PLK-WT, and PLK-GFP $\left(5 \times 10^{6}\right)$ were incubated in $100 \mu l$ of GIT medium. The culture supernatants were collected at 15 and 30 $\mathrm{min}$. The samples were incubated in 96-well plates coated with rabbit anti-rTgPDCD5 polyclonal $\mathrm{IgG}$, and detected with mouse anti-TgPDCD5 serum and horseradish peroxidase-conjugated goat anti-mouse IgG antibodies. After reaction with the substrate solution, the absorbance at $415 \mathrm{~nm}$ was measured. The TgPDCD5 concentration was calculated by standardization with rTgPDCD5. The line graph indicates the mean and S.D. of triplicate samples. Statistical significance against PLKWT was calculated using Student's $t$-test.

a mechanism of immune evasion adopted by the parasite, through suppression of antigen presenting cells [11, 25]. Hence, inhibition of both antigen presentation by the antigen-presenting cells and antigen-recognition by lymphocytes may be implicated in the reduced immune response caused by $T$. gondii-infection.

In our previous study, apoptosis enhancement of parasiteinfected macrophages by recombinant proteins and IFN- $\gamma$ showed that the rate of apoptosis reflected the amount of TgPDCD5 in the medium [1]. This observation was consistent with the results from cells treated with different doses of rTgPDCD5 and with etoposide [1]. The apoptosis assay using the overexpressing parasite also reflected TgPDCD5 dose-dependency. However, significantly increased apoptosis was only observed after PLK-TgPDCD5 infection, but not after PLK- $\Delta 106$ infection, suggesting the necessity for the HSPG-binding motif, as observed in the assay using recombinant proteins [1]. This may be due to the fact that the overproduced $\Delta 106-122$ protein, lacking the motif required for cell penetration, was not internalized by uninfected cells.

It was also notable that only pico-molar levels of native TgPDCD5 were detected in the culture supernatant at $24 \mathrm{hr}$ after the infection. The concentration of secreted TgPDCD5 in the PLK-TgPDCD5-1-, PLK-TgPDCD5-2- and PLK-

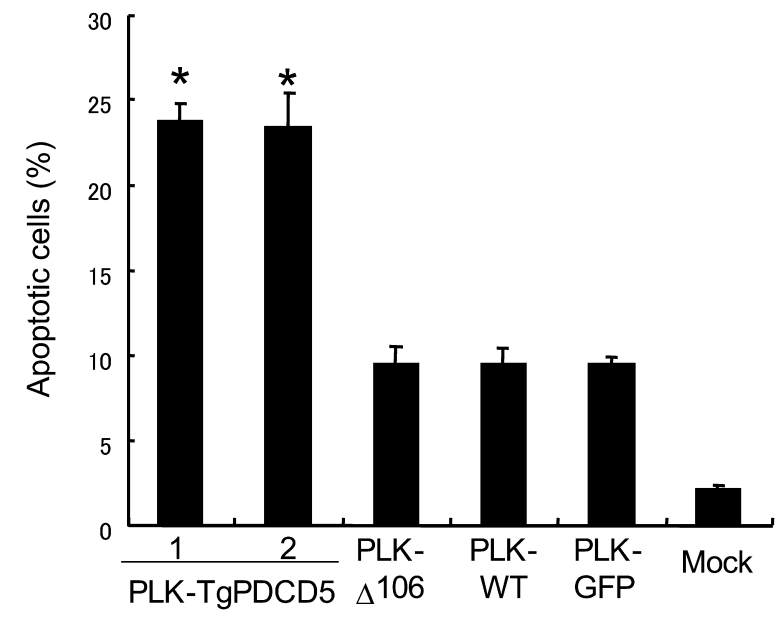

Fig. 3. Evaluation of the effects of PLK-TgPDCD5 on host cell apoptosis in the presence of interferon (IFN) $-\gamma$. J774A. 1 cells ( 5 $\times 10^{5}$ ) were inoculated with $T$. gondii PLK-TgPDCD5-1, PLKTgPDCD5-2, PLK- $\Delta 106$, PLK-WT, and PLK-GFP $\left(5 \times 10^{5}\right)$ and incubated with $20 \mathrm{U} / \mathrm{m} l \mathrm{IFN}-\gamma$. After treatment, genomic DNA fragmentation was detected by TUNEL staining, and the ratio of apoptotic cells was determined. The histograms indicate the mean and S.D. of triplicate samples. Statistical significance against PLK-WT was calculated using the Student's $t$ test. $* P<0.01$.

$\Delta$ 106-infected groups were $4.00 \pm 0.46 \mathrm{pg} / \mathrm{ml}(P<0.01)$, $4.37 \pm 0.91 \mathrm{pg} / \mathrm{m} l(P<0.01)$ and $3.67 \pm 0.42 \mathrm{pg} / \mathrm{m} l(P<0.01)$, respectively. These were significantly higher than those in the PLK-WT- $(1.43 \pm 0.42 \mathrm{pg} / \mathrm{m} l)$, and PLK-GFP-infected $(0.97 \pm 0.06 \mathrm{pg} / \mathrm{m} l)$ groups. Since the ratios of apoptotic cells observed in the PLK-TgPDCD5-1- and PLKTgPDCD5-2-infected groups were significantly higher than those in the PLK-WT- and PLK-GFP-infected groups, supernatant of parasite cultures containing pico-molar level of native TgPDCD5 can enhance host-cell apoptosis. However, the level of recombinant protein that produced apoptosis enhancement in the previous study was in the micromolar order. This might be due to the difference in expression origins; it is possible that rTgPDCD5 expressed in $E$. coli might possess a lower apoptosis-enhancing activity than parasite-expressed native protein. Alternatively, the pro-apoptotic effect of this molecule alone may not be strong enough to induce apoptosis; it may require other, additional factors. Further studies are needed to identify such as yet unknown factor(s). The effects of TgPDCD5 on other kinds of immune cells and its involvement in the apoptosis observed in various tissues during parasite infection should be also addressed in future studies.

A sandwich ELISA successfully detected a time-dependent increase (after $15 \mathrm{~min}$ ) in TgPDCD5 protein levels in the culture medium of free parasites. We suggested that TgPDCD5 secreted from the extracellular tachyzoites was internalized by host cells before the invasion and by the uninfected host cells. Interestingly, treatment of J774A.1 cells with rTgPDCD5 protein and IFN- $\gamma$ showed no 
enhancement of host-cell apoptosis in T. gondii-infected cells while apoptosis was enhanced in the uninfected cells (1). Since $T$. gondii-infected host cells are resistant against apoptosis induction by interference with the caspase cascade, increased expression of antiapoptotic molecules by infected host cells, and a decreased activity of the poly(ADP-ribose) polymerase (19), effects of TgPDCD5 might be cancelled in the infected cells. In conclusion, endogenous TgPDCD5 protein was active in enhancing host cell apoptosis, as previously reported for recombinant TgPDCD5. The involvement of the HSPG-binding motif was also confirmed, suggesting that penetration of this molecule into the host cells is essential for its activity. However, the mechanism of TgPDCD5 function remains unclear. Further studies are required to elucidate the mechanisms whereby TgPDCD5 stimulates apoptosis in $T$. gondii-infected cells or animals.

ACKNOWLEDGMENTS. We thank Dr. K.A. Joiner (Yale Univ.) for providing the plasmid vector $\mathrm{pHXNTPHA.} \mathrm{This}$ study was supported by Grants-in-Aid for Scientific Research on Priority Areas (19041008), the Ministry of Education, Culture, Sports, Science and Technology of Japan. The first author has been supported by Research Fellowships of the Japan Society for the Promotion of Science for Young Scientists (19-21).

\section{REFERENCES}

1. Bannai, H., Nishikawa, Y., Matsuo, T., Kawase, O., Watanabe, J., Sugimoto, C. and Xuan, X. 2008. Programmed Cell Death 5 from Toxoplasma gondii: A secreted molecule that exerts a pro-apoptotic effect on host cells. Mol. Biochem. Parasitol. 159: $112-120$.

2. Chen, Y. Y., Sun, R. H., Han, W. L., Zhang, Y., Song, Q., Di, C. and Ma, D. 2001. Nuclear translocation of PDCD5 (TFAR19): an early signal for apoptosis? FEBS Lett. 509: 191196.

3. Dubey, J. P. and Beattie, C. P. 1988. Toxoplasmosis of animals and man, CRC Press, Boca Ranton.

4. Elliott, G. and O'Hare, P. 1997. Intracellular trafficking and protein delivery by a herpesvirus structural protein. Cell $\mathbf{8 8}$ : 223-233.

5. Frankel, A. D. and Pabo, C. O. 1988. Cellular uptake of the tat protein from human immunodeficiency virus. Cell 55: 1189 1193.

6. Gavrilescu, L. C. and Denkers, E. Y. 2001. IFN- $\gamma$ overproduction and high level apoptosis are associated with high but not low virulence Toxoplasma gondii infection. J. Immunol. 167: 902-909.

7. Gavrilescu, L. C. and Denkers, E. Y. 2003. Interleukin-12 p40and Fas ligand-dependent apoptotic pathways involving STAT-1 phosphorylation are triggered during infection with a virulent strain of Toxoplasma gondii. Infect. Immun. 71: 25772583.

8. Green, M. and Loewenstein, P. M. 1988. Autonomous functional domains of chemically synthesized human immunodeficiency virus tat trans-activator protein. Cell 55: 1179-1188.

9. Guha, M., Choubey, V., Maity, P., Kumar, S., Shrivastava, K., Puri, S. K. and Bandyopadhyay, U. 2007. Overexpression, purification and localization of apoptosis related protein from Plasmodium falciparum. Protein Expr. Purif. 52: 363-372.

10. Heussler, V. T., Kuenzi, P. and Rottenberg, S. 2001. Inhibition of apoptosis by intracellular protozoan parasites. Int. J. Parasitol. 31: 1166-1176.

11. Hisaeda, H., Sakai, T., Ishikawa, H., Maekawa, Y., Yasutomo, K., Good, R. A. and Himeno, K. 1997. Heat shock protein 65 induced by $\gamma \delta \mathrm{T}$ cells prevents apoptosis of macrophages and contributes to host defense in mice infected with Toxoplasma gondii. J. Immunol. 159: 2375-2381.

12. Joliot, A., Pernelle, C., Deagostine-Bazin, H. and Prochiantz, A. 1991. Antennapedia homeobox peptide regulates neural morphogenesis. Proc. Natl. Acad. Sci. U.S.A. 88: 1864-1868.

13. Khan, A., Böhme, U., Kelly, K. A., Adlem, E., Brooks, K., Simmonds, M., Mungall, K., Quail, M. A., Arrowsmith, C., Chillingworth, T., Churcher, C., Harris, D., Collins, M., Foster, N., Fraser, A., Hance, Z., Jagels, K., Moule, S., Murphy, L., O̊̊fNeil, S., Rajandream, M. A., Saunders, D., Seeger, K., Whitehead, S., Mayr, T., Xuan, X., Watanabe, J., Suzuki, Y., Wakaguri, H., Sugano, S., Sugimoto, C., Paulsen, I., Mackey, A. J., Roos, D. S., Hall, N., Berriman, M., Barrell, B., Sibley, L. D. and Ajioka, J. W. 2006. Common inheritance of chromosome Ia associated with clonal expansion of Toxoplasma gondii. Genome Res. 16: 1119-1125.

14. Khan, I. A., Matsuura, T. and Kasper, L. H. 1996. Activationmediated $\mathrm{CD}^{+} \mathrm{T}$ cell unresponsiveness during acute Toxoplasma gondii infection in mice. Int. Immunol. 8: 887-896.

15. Lang, C., Algner, M., Beinert, N., Gross, U. and Lüder, C. G. K. 2006. Diverse mechanisms employed by Toxoplasma gondii to inhibit IFN- $\gamma$-induced major histocompatibility complex class II gene expression. Microbes Infect. 8: 1994-2005.

16. Lieberman, J. 2003. The ABCs of granule-mediated cytotoxicity: new weapons in the arsenal. Nat. Rev. Immunol. 3: 361370 .

17. Liesenfeld, O., Kosek, J. C. and Suzuki, Y. 1997. Gamma interferon induces Fas-dependent apoptosis of Peyer's patch T cells in mice following peroral infection with Toxoplasma gondii. Infect. Immun. 65: 4682-4689.

18. Liu, H. T., Wang, Y. G., Zhang, Y. M., Song, Q., Di, C., Chen, G., Tang, J. and Ma, D. 1999. TFAR19, a novel apoptosisrelated gene cloned from human leukemia cell line TF-1, could enhance apoptosis of some tumor cells induced by growth factor withdrawal. Biochem. Biophys. Res. Commun. 254: 203210.

19. Lüder, C. G. K. and Gross, U. 2005. Apoptosis and its modulation during infection with Toxoplasma gondii: molecular mechanisms and role in pathogenesis. Curr. Top. Microbiol. Immunol. 289: 219-237.

20. Lüder, C. G. K., Gross, U. and Lopes, M. F. 2001. Intracellular protozoan parasites and apoptosis: diverse strategies to modulate parasite-host interactions. Trends. Parasitol. 17: 480-486.

21. Lüder, C. G. K., Lang, T., Beuerle, B. and Gross, U. 1998. Down-regulation of MHC class II molecules and inability to up-regulate class I molecules in murine macrophages after infection with Toxoplasma gondii. Clin. Exp. Immunol. 112: 308-316.

22. Luft, B. J., Kansas, G., Engleman, E. G. and Remington, J. S. 1984. Functional and quantitative alterations in T lymphocyte subpopulations in acute toxoplasmosis. J. Infect. Dis. 150: 761-767.

23. Luft, B. J. and Remington, J. S. 1988. AIDS commentary. Toxoplasmic encephalitis. J. Infect. Dis. 157: 1-6.

24. Mordue, D. G., Monroy, F., La Regina, M., Dinarello, C. A. 
and Sibley, L. D. 2001. Acute toxoplasmosis leads to lethal overproduction of Th1 cytokines. J. Immunol. 167: 4574-4584.

25. Nishikawa, Y., Kawase, O., Vielemeyer, O., Joiner, K. A., Xuan, X. and Nagasawa, H. 2007. Toxoplasma gondii infection induces apoptosis in noninfected macrophages: role of nitric oxide and other soluble factors. Parasite Immunol. 29: 375385.

26. Nishikawa, Y., Xuan, X., Makala, L., Vielemeyer, O., Joiner, K. A. and Nagasawa, H. 2003. Characterization of Toxoplasma gondii engineered to express mouse interferon-gamma. Int. J. Parasitol. 33: 1525-1535.

27. Nishikawa, Y., Zhang, H., Ibrahim, H. M., Ui, F., Ogiso, A. and Xuan, X. 2008. Construction of Toxoplasma gondii bradyzoite expressing the green fluorescent protein. Parasitol. Int. 57: 219-222.

28. Opferman, J. T. and Korsmeyer, S. J. 2003. Apoptosis in the development and maintenance of the immune system. Nat. Immunol. 4: 410-415.

29. Remington, J. S. and Desmonts, G. 1990. Toxoplasmosis. pp. 89-195. In: Infectious Diseases of the Fetus and Newborn Infant, 3rd ed. (Remington, J. S. and Klein, J. O. eds.), WB Saunders, Philadelphia.

30. Rui, M., Chen, Y. Y., Zhang, Y. M. and Ma, D. L. 2002. Transfer of anti-TFAR19 monoclonal antibody into HeLa cells by in situ electroporation can inhibit the apoptosis. Life Sci. 71: 1771-1778.

31. Sibley, L. D., Messina, M. and Niesman, I. R. 1994. Stable DNA transformation in the obligate intracellular parasite Toxoplasma gondii by complementation of tryptophan auxotrophy. Proc. Natl. Acad. Sci. U.S.A. 91: 5508-5512.
32. Strickland, G. T. and Sayles, P. C. 1977. Depressed antibody responses to a thymus-dependent antigen in toxoplasmosis. Infect. Immun. 15: 184-190.

33. Tenter, A. M., Heckeroth, A. R. and Weiss, L. M. 2000. Toxoplasma gondii: From animal to humans. Int. J. Parasitol. 30: 1217-1258.

34. Wang, Y., Li, D., Fan, H., Tian, L., Zhong, Y., Zhang, Y., Yuan, L., Jin, C., Yin, C. and Ma, D. 2006. Cellular uptake of exogenous human PDCD5 protein. J. Biol. Chem. 281: 24803 24817.

35. Wang, Y., Li, X. T., Wang, L., Ding, P., Zhang, Y., Han, W. and Ma, D. 2004. An alternative form of paraptosis-like cell death, triggered by TAJ/TROY and enhanced by PDCD5 overexpression. J. Cell Sci. 117: 1525-1532.

36. Wei, S., Marches, F., Borvak, J., Zou, W., Channon, J., White, M., Radke, J., Cesbron-Delauw, M. F. and Curiel, T. J. 2002. Toxoplasma gondii-infected human myeloid dendritic cells induce T-lymphocyte dysfunction and contact-dependent apoptosis. Infect. Immun. 70: 1750-1760.

37. Williams, G. T. 1994. Programmed cell death: a fundamental protective response to pathogens. Trends Microbiol. 2: 463464.

38. Wing, E. J., Boehmer, S. M. and Christner, L. K. 1983. Toxoplasma gondii: decreased resistance to intracellular bacteria in mice. Exp. Parasitol. 56: 1-8.

39. Yano, A., Norose, K., Yamashita, K., Aosai, F., Sugane, K., Segawa, K. and Hayashi, S. 1987. Immune response to Toxoplasma gondii-analysis of suppressor T cells in a patient with symptomatic acute toxoplasmosis. J. Parasitol. 73: 954-961. 